\title{
HER2 Dimerization Inhibitor Pertuzumab - Mode of Action and Clinical Data in Breast Cancer
}

\author{
Nadia Harbeck ${ }^{a}$ Matthias W. Beckmann ${ }^{b}$ Achim Rody ${ }^{c}$ Andreas Schneeweiss $^{d}$ \\ Volkmar Müller ${ }^{\mathrm{e}} \quad$ Tanja Fehm $^{f} \quad$ Norbert Marschner $^{\mathrm{g}} \quad$ Oleg Gluz $^{\mathrm{h}} \quad$ Iris Schrader $^{\mathrm{i}}$ \\ Georg Heinrich ${ }^{j}$ Michael Untch ${ }^{k}$ Christian Jackisch'
}

\begin{abstract}
aBrustzentrum, Frauenklinik, Universität München (LMU), 'brauenklinik, Universität Erlangen,
'Klinik für Frauenheilkunde und Geburtshilfe Lübeck, Universitätsklinikum Schleswig-Holstein,

${ }^{d}$ Nationales Centrum für Tumorerkrankungen, Universitätsklinikum Heidelberg,

${ }^{\mathrm{e}}$ Klinik und Poliklinik für Gynäkologie, Universitätsklinikum Hamburg-Eppendorf, ${ }^{\mathrm{f}}$ Frauenklinik, Universitätsklinikum Tübingen,

9Praxis für Interdisziplinäre Onkologie und Hämatologie, Freiburg i.Br.,

hWestdeutsche Studiengruppe GmbH, Brustzentrum Niederrhein, Evangelisches Krankenhaus Bethesda, Mönchengladbach,

'Gynäkologisch-Onkologische Schwerpunktpraxis, Hannover, ${ }^{\mathrm{i} G y n a ̈ k o l o g i s c h-O n k o l o g i s c h e ~ S c h w e r p u n k t p r a x i s, ~ F u ̈ r s t e n w a l d e, ~}$

kKlinik für Gynäkologie, HELIOS Klinikum Berlin-Buch, 'Klinik für Gynäkologie und Geburtshilfe, Klinikum Offenbach, Germany
\end{abstract}

\section{Keywords}

HER2-positive · Dual inhibition · Breast cancer, metastatic .

Pertuzumab · Trastuzumab

\section{Summary}

The humanized monoclonal antibody pertuzumab prevents the dimerization of HER2 with other HER receptors, in particular the pairing of the most potent signaling heterodimer HER2/HER3, thus providing a potent strategy for dual HER2 inhibition. It binds to the extracellular domain of HER2 at a different epitope than trastuzumab. Pertuzumab and trastuzumab act in a complementary fashion and provide a more complete blockade of HER2-mediated signal transduction than either agent alone. Phase II studies demonstrated that pertuzumab was generally well tolerated as a single agent or in combination with trastuzumab and/or cytotoxic agents, and implied an improved clinical efficacy of the combination of pertuzumab and trastuzumab in early and advanced HER2-positive breast cancer. Results of the pivotal phase III study CLEOPATRA in patients with HER2-positive metastatic breast cancer demonstrated that the addition of pertuzumab to first-line combination therapy with docetaxel and trastuzumab significantly prolonged progression-free and overall survival without increasing cardiac toxicity. Currently, the combination of both antibodies is being explored in the palliative setting as well as in the treatment of early HER2positive breast cancer. Dual HER2 inhibition with the HER2 dimerization inhibitor pertuzumab and trastuzumab may change clinical practice in HER2-positive first-line metastatic breast cancer treatment.

\section{Schlüsselwörter}

HER2-positiv · Duale Inhibition · Mammakarzinom, metastasiertes · Pertuzumab · Trastuzumab

\section{Zusammenfassung}

Der humanisierte monoklonale Antikörper Pertuzumab verhindert die Dimerisierung von HER2 mit anderen HERRezeptoren, insbesondere die Bildung des im Hinblick auf Signaltransduktionskaskaden potentesten Heterodimers HER2-HER3 und ermöglicht damit eine grundlegend andere Strategie der dualen HER2-Inhibition. Pertuzumab bindet an die extrazelluläre Domäne von HER2, aber einem anderen Epitop als Trastuzumab. Pertuzumab und Trastuzumab erreichen zusammen eine vollständigere Blockade der HER2vermittelten Signaltransduktion als jede Substanz alleine. Phase-II-Studien konnten zeigen, dass Pertuzumab alleine oder in Kombination gut verträglich ist. Sie legten auch eine verbesserte klinische Wirksamkeit der Kombination von Pertuzumab und Trastuzumab bei primärem und fortgeschrittenem HER2-positiven Brustkrebs nahe. Die Ergebnisse der Phase-III-Studie CLEOPATRA bei Patientinnen mit metastasiertem HER2-positivem Mammakarzinom zeigten, dass die zusätzliche Gabe von Pertuzumab zu einer Erstlinien-Kombinationstherapie mit Docetaxel und Trastuzumab das progressionsfreie Überleben und das Gesamtüberleben signifikant verlängerte ohne Steigerung der kardialen Toxizität. Derzeit wird die Kombination aus beiden Antikörpern in der palliativen Situation und in der Behandlung des frühen HER2-positiven Mammakarzinoms untersucht. Die duale HER2-Inhibition mit Trastuzumab und dem HER2 Dimerisationsinhibitor Pertuzumab könnte die klinische Praxis für die Erstlinientherapie beim metastasierten HER2-positiven Mammakarzinom und möglicherweise auch in früheren Stadien verändern.

\section{KARGER}

Fax +497614520714

Information@Karger.com

www.karger.com
(C) 2013 S. Karger GmbH, Freiburg

$1661-3791 / 13 / 0081-0049 \$ 38.00 / 0$

Accessible online at:

www.karger.com/brc 


\section{Introduction}

According to a current analysis of more than 18,000 results of HER2 testing in clinical routine practice, $16.7 \pm 3.2 \%(95 \%$ confidence interval (CI) 16.6-16.8) of patients with early breast cancer have HER2-positive tumors [1]. HER2 positivity is correlated with aggressive breast tumor behavior and associated with reduced response to standard therapies and decreased breast cancer survival [2, 3]. The introduction of targeted therapy against HER2 has significantly changed the prognosis of patients with HER2-positive breast cancer, transforming HER2 positivity from a negative prognostic to a positive predictive disease marker. Trastuzumab has become the standard of care that significantly improves outcomes in patients with HER2-positive early breast cancer [4-9] and HER2-positive metastatic breast cancer (MBC) [10, 11]. Yet, despite the proven efficacy of trastuzumab plus chemotherapy, some patients with HER2-positive breast cancer do not respond, and the disease in the majority of patients with MBC progresses within 1 year. The medical need for targeted therapy for advanced disease is being addressed through intensive research efforts. Dual therapy targeting HER2 has been identified as a promising treatment strategy [12]. The humanized monoclonal antibody pertuzumab binds to the extracellular domain of HER2. It prevents the dimerization of HER2 with other HER receptors, especially HER3 [13] and allows for a fundamentally different strategy of dual HER2 inhibition.

\section{HER Family and HER2-HER3 Heterodimer}

The 4 type 1 transmembrane tyrosine kinase receptors EGFR (or HER1), HER2, HER3 and HER4 constitute the HER family. HER1, HER2 and HER3 are all implicated in the development and progression of cancer [14]. In principle, each receptor consists of an extracellular domain that binds ligands, a transmembrane region and a cytoplasmic domain with kinase activity, with the exceptions that there is no known ligand to HER2, and HER3 does not exert tyrosine kinase activity. Ligand binding induces conformational rearrangements of the receptors, and promotes association of both homodimers and heterodimers. This is followed by internalization, phosphorylation and further downstream signaling, thus regulating many cellular processes including cell growth, proliferation and survival [15].

Not all of the 10 possible dimers are fully biologically active. HER2 and HER3 are highly complementary to each other: HER3 binds ligand yet lacks intrinsic kinase activity; HER2 has intrinsic tyrosine kinase activity but no identified ligand [14]. The HER2/HER3 heterodimer is considered the most potent HER dimer pair with respect to strength of interaction, ligand-induced tyrosine phosphorylation and downstream signaling $[16,17]$. In vitro, reduction of HER3 expres-

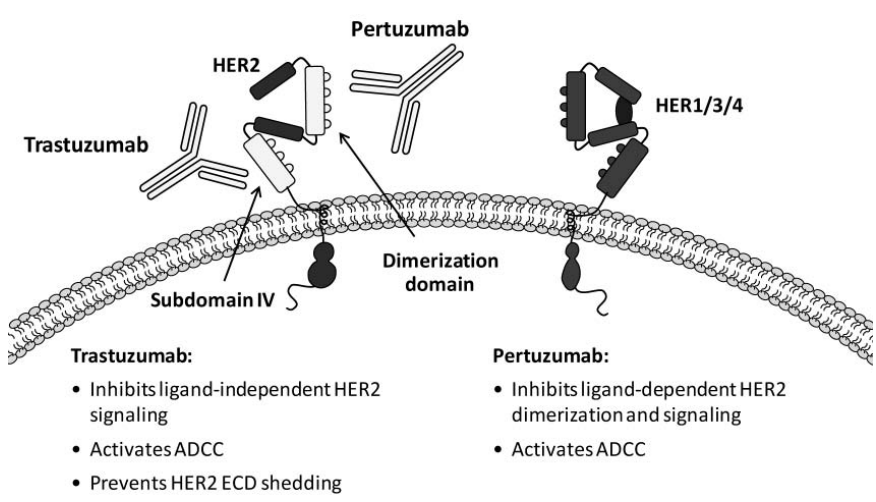

Fig. 1. Complementary mechanisms of action of pertuzumab and trastuzumab. Adapted from Baselga J et al. [40] ADCC = antibody-dependent cell-mediated cytotoxicity; $\mathrm{ECD}=$ extracellular domain

sion decreased cell proliferation to the same extent as reduction of HER2 expression [18]. Also, HER3 provides an escape route for breast cancer when HER1 and HER2 are inhibited by tyrosine kinase inhibitors [19, 20].

\section{Pertuzumab - Mode of Action and Rationale for Combination with Trastuzumab}

Pertuzumab is a humanized monoclonal antibody that binds to the dimerization domain of HER2. Blocking the pairing of the most potent signaling HER dimer, HER2-HER3, affects key signaling pathways $[13,17,20,21]$. As it binds to the extracellular domain of HER2 pertuzumab can also activate immune effector functions such as antibody-dependent cellmediated cytotoxicity [22].

In vitro studies have shown that trastuzumab and pertuzumab bind to different epitopes on the HER2 protein [13]. Concomitant binding of both antibodies has also been demonstrated in vivo [22]. The modes of action of trastuzumab and pertuzumab are complementary (fig. 1). Trastuzumab inhibits ligand-independent HER2 signaling, prevents HER2 activation by extracellular domain shedding, and flags cells for destruction by the immune system [22-24]; however, it cannot prevent ligand-activated HER2/HER3 or HER2/ HER1 heterodimerization, a potential escape mechanism for tumor cells from the inhibitory effects of trastuzumab [13,21, 25]. There is preclinical evidence for the synergy of trastuzumab and pertuzumab in HER2-overexpressing tumors and specifically in HER2-overexpressing breast cancer. It was demonstrated that trastuzumab and pertuzumab synergistically inhibited the survival of HER2-overexpressing BT474 breast cancer cells in vitro [24]. In vivo, the combination of trastuzumab and pertuzumab dramatically enhanced the antitumor effect in an HER2-overexpressing breast cancer xenograft model compared to each antibody as a single agent [22]. 
In addition, the combination of pertuzumab and trastuzumab was effective following progression on trastuzumab [22].

\section{Clinical Data}

\section{Monotherapy with Pertuzumab}

Single-agent pertuzumab has been evaluated in 2 phase I studies in 39 patients with solid tumors. The results demonstrated that pertuzumab was well tolerated and had a pharmacokinetic profile supporting 3-week dosing [26]. The maximum tolerated dose (MTD) was not reached up to the dose level of $25 \mathrm{mg} / \mathrm{kg}$ [27]. Results also implied potential flat dosing.

Five phase II studies in unselected patients with advanced prostate, non-small cell, ovarian and breast cancer demonstrated a modest clinical activity of single-agent pertuzumab [28-32]. Pertuzumab was generally well tolerated. Most adverse events were grade 1-2. Diarrhea, rash, asthenia, vomiting, nausea and abdominal pain were most common. A pooled analysis of 598 patients from 14 studies with pertuzumab as a single agent, or in combination with capecitabine, docetaxel, gemcitabine, carboplatin, paclitaxel, erlotinib or trastuzumab showed an at least similar cardiac safety as trastuzumab. 23 of 331 patients across all studies with singleagent pertuzumab developed asymptomatic left ventricular systolic dysfunction and $1(0.3 \%)$ symptomatic heart failure [33]. There was no notable increase in cardiac side effects when pertuzumab was given in combination with non-anthracycline cytotoxics, erlotinib or trastuzumab.

A phase II study performed in 79 patients with HER2negative breast cancer evaluated pertuzumab given once every 3 weeks with a loading dose of $840 \mathrm{mg}$ followed by either $420 \mathrm{mg}$ or $1,050 \mathrm{mg}$. Pharmacokinetic data supported a fixed dose of pertuzumab once every 3 weeks [32]. Analysis of pooled data from 153 patients from 3 clinical studies in which pertuzumab was either administered every 3 weeks as a weight-based or fixed dose (420 or $1,050 \mathrm{mg}$ ) indicated that pharmacokinetic profiles were similar, thus proving the feasibility of administering pertuzumab as a fixed dose [34].

\section{Dual HER2 Inhibition with Pertuzumab and Trastuzumab- Phase II Studies}

A phase II study in patients with HER2-positive MBC with progression during prior trastuzumab therapy provided the first evidence that the combination of the 2 antibodies pertuzumab and trastuzumab, without additional chemotherapy, was clinically active and well tolerated [35]. The overall response rate for the 66 patients treated with the combination of both antibodies was $24.2 \%$. Complete response (CR) was seen in $4(6.1 \%)$, partial response (PR) in $12(18.2 \%)$, and stable disease (SD) for 6 months in $17(25.8 \%)$ patients. Median progression-free survival (PFS) was 5.5 months. Adverse events were generally grade 1 or 2 and included diarrhea, fatigue, nausea, and rash. 4 patients experienced treatment-related adverse events of grade 3 or higher. There were no significant cardiac events. Only 3 patients had a decrease in left ventricular ejection fraction (LVEF) of $>10 \%$ points from baseline or to $<50 \%$ of the absolute value. No patient withdrew due to cardiac-related adverse events [35].

Data for the efficacy and safety of the combination of pertuzumab and trastuzumab also come from 2 large randomized studies in the neoadjuvant setting. 417 patients with locally advanced, inflammatory or early HER2-positive breast cancer were enrolled in the international randomized phase II trial NeoSphere [36]. Patients were randomized into 4 arms and received trastuzumab plus docetaxel in arm A, pertuzumab, trastuzumab plus docetaxel in arm B, pertuzumab and trastuzumab in arm $\mathrm{C}$ and pertuzumab plus docetaxel in arm D. Study drugs were given every 3 weeks for 4 cycles before surgery. Trastuzumab was given with a loading dose of $8 \mathrm{mg} / \mathrm{kg}$ and at $6 \mathrm{mg} / \mathrm{kg}$ subsequently, pertuzumab at a loading dose of $840 \mathrm{mg}$ and then at $420 \mathrm{mg}$, and docetaxel at $75 \mathrm{mg} / \mathrm{m}^{2}$ escalating if tolerated to $100 \mathrm{mg} / \mathrm{m}^{2}$. The highest pathological CR (pCR) of $45.8 \%$ was achieved with the combination of pertuzumab, trastuzumab plus docetaxel as compared to $29 \%$ for trastuzumab plus docetaxel $(\mathrm{p}=0.0141)$ and $24.0 \%$ with pertuzumab plus docetaxel $(\mathrm{p}=0.003)$. Of note, patients treated with the chemotherapy-free combination of pertuzumab and trastuzumab had a pCR of $16.8 \%$. Fewer pCRs were seen in hormone receptor-positive tumors across all study arms. The most frequent adverse events were mostly grade 1-2 and were alopecia, neutropenia, diarrhea, nausea, fatigue rash and mucosal inflammation. The lowest incidence of adverse events $\geq$ grade 3 was recorded for the combination of pertuzumab and trastuzumab. The mean maximum decrease in LVEF was low (4-5\%) and balanced across treatment arms. Adding pertuzumab to trastuzumab did not result in an increase of cardiac toxicity [36]. Extensive biomarker analyses have been performed but so far have not yielded clinically useful assays for patient or regimen selection in addition or alternatively to conventional HER2 assessment used at present [37].

Another 223 patients with HER2-positive locally advanced, inflammatory or early stage breast cancer were assigned to 3 arms of 6 cycles of neoadjuvant treatment in the TRYPHAENA study, an international randomized phase II study [38]. Patients in arm A received FEC (5-flurouracil, epirubicin, cyclophosphamide) in cycles 1-3 and docetaxel in cycles 4-6 plus pertuzumab and trastuzumab from cycles 1-6. Patients in arm B were treated with FEC from cycles 1-3 and docetaxel from cycles 4-6 plus pertuzumab and trastuzumab from cycles 4-6 only. In arm $\mathrm{C}$, patients received docetaxel and carboplatin plus pertuzumab and trastuzumab from cycles 1-6. Study drugs were administered every 3 weeks. Pertuzumab was given at a loading dose of $840 \mathrm{mg}$ and at $420 \mathrm{mg}$ subsequently, and trastuzumab at a loading dose of $8 \mathrm{mg} / \mathrm{kg}$ and at $6 \mathrm{mg} / \mathrm{kg}$ subsequently. Following surgery, patients received trastuzumab to complete 1 year of treatment. The primary endpoint 
of the study was cardiac safety, assessed by a thorough followup of $15 \mathrm{LVEF}$ assessments up to 60 months post randomization. Cardiac toxicity was generally mild in all treatment arms with symptomatic left ventricular systolic dysfunction (LVSD) $\geq$ grade 3 in $0 \%, 2.7 \%$ and $0 \%$, and LVEF decline $\geq 10 \%$ points from baseline or to $<50 \%$ in $4.2 \%, 5.3 \%$ and $3.9 \%$ of patients for arms A, B, and C, respectively. There was no difference in cardiac side effects for concurrent administration of pertuzumab plus trastuzumab with epirubicin compared with sequential administration or the anthracycline-free regimen. Neutropenia, febrile neutropenia, leukopenia, and diarrhea were most frequently reported adverse events of $\geq$ grade 3 across all arms. Regardless of chemotherapy chosen, the combination of pertuzumab with trastuzumab in the neoadjuvant setting resulted in high pCR rates of $61.6 \%, 57.3 \%$ and $66.2 \%$ for arms A, B, and C, respectively [38].

\section{Dual HER2 Inhibition with Pertuzumab and Trastuzumab- Pivotal Phase III Study in First-line MBC Treatment}

In the pivotal phase III trial CLEOPATRA, 808 patients with centrally confirmed HER2-positive locally recurrent, unresectable, or metastatic breast cancer were randomized $[39,40]$. For inclusion, patients could have had only a prior hormonal regimen for MBC. Prior neoadjuvant or adjuvant systemic therapy, including trastuzumab and/or taxanes, was allowed if the disease-free interval was $\geq 12$ months. Patients were treated until the time of disease progression or the development of unacceptable toxicity either with a combination of docetaxel, trastuzumab and placebo in the control group or docetaxel, trastuzumab and pertuzumab in the experimental arm (fig. 2). The recommendation was to administer at least 6 cycles of chemotherapy. If chemotherapy was discontinued due to toxicity, antibody therapy could be continued until disease progression or onset of unacceptable toxicity. $75 \%$ of all patients discontinued docetaxel but continued to receive pertuzumab or placebo plus trastuzumab treatment. The median number of treatment cycles per patient was 15 (range 1-50) in the control and 18 (range 1-56) in the pertuzumab

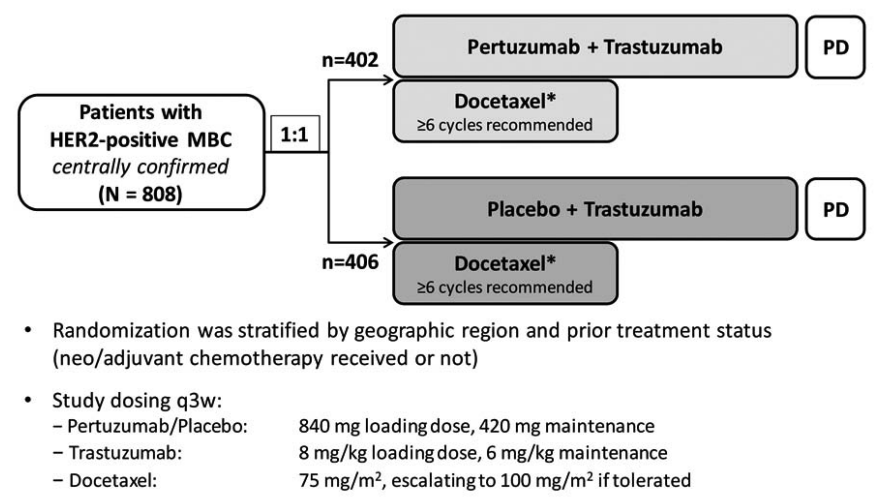

Fig. 2. CLEOPATRA: Study design. Adapted from Baselga J et al. [40]. $\mathrm{MBC}=$ metastatic breast cancer $\mathrm{PD}=$ progressive disease.

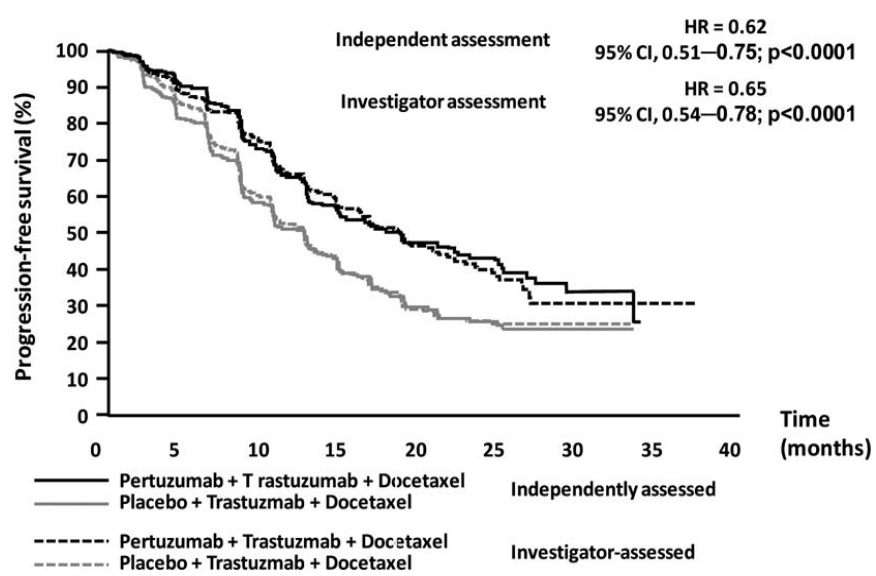

Fig. 3. Independently and investigator-assessed progression-free survival. Adapted from Baselga $\mathbf{J}$ et al. [39, 40].

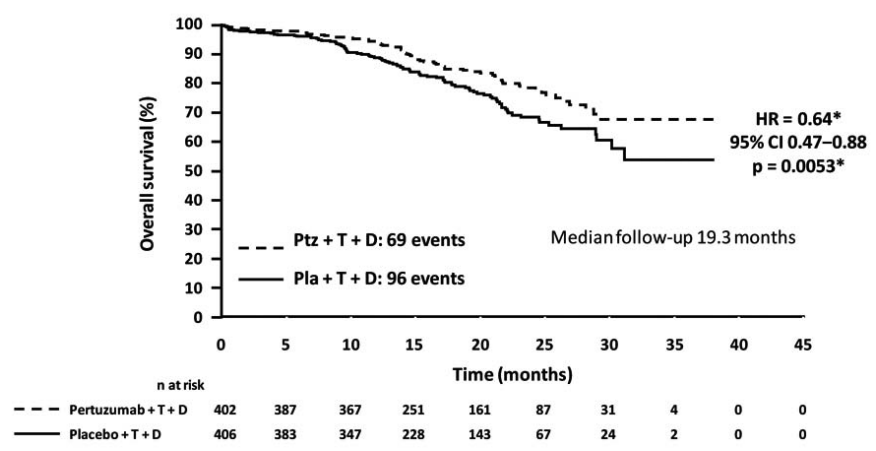

Fig. 4. CLEOPATRA: Predefined interim analysis of overall survival $(n=165$ events). Adapted from Baselga $J$ et al. $[39,40]$.

$\mathrm{D}=$ docetaxel; $\mathrm{OS}=$ overall survival $; \mathrm{Pla}=$ placebo Ptz = pertuzumab; $\mathrm{T}=$ trastuzumab.

group. Median duration of study treatment was 11.8 and 18.1 months, respectively. A median of 8 cycles of docetaxel were administered in both groups.

The primary endpoint of independently assessed PFS was met. Pertuzumab increased PFS by 6.1 months from 12.4 months in the control group to 18.5 months in the experimental arm (fig. 3). The treatment benefit was consistent across all prespecified subgroups except for the subgroup of patients with non-visceral disease. In particular, there was a clear treatment benefit in both hormone receptor-negative and -positive patients. In the 288 patients who had received adjuvant or neoadjuvant therapy without trastuzumab, independently assessed PFS was 12.6 months for the control and 21.6 months for the pertuzumab group (hazard ratio $(\mathrm{HR})=$ 0.60 ; $95 \%$ CI, $0.43-0.83$ ). For the 88 patients with prior neoadjuvant or adjuvant trastuzumab, median independently assessed PFS was 10.4 and 16.9 months $(\mathrm{HR}=0.62 ; 95 \% \mathrm{CI}$, 0.35-1.07), respectively.

Analysis of the secondary endpoint, overall survival, is event-driven. A preplanned interim analysis performed after 165 events and a median follow-up of 19.3 months showed a 
Table 1. CLEOPATRA: Cardiac tolerability

\begin{tabular}{|c|c|c|}
\hline & Placebo + trastuzumab + docetaxel & Pertuzumab + trastuzumab + docetaxel \\
\hline $\mathrm{n}$ & 397 & 407 \\
\hline Investigator-assessed symptomatic LVSD ${ }^{\mathrm{b}}$ & $1.8 \%$ & $1.0 \%$ \\
\hline Independently adjudicated symptomatic LVSD & $1.0 \%$ & $1.0 \%$ \\
\hline Fall in LVEF to $<50 \%$ or by $\geq 10 \%$ points from baseline & $6.6 \%$ & $3.8 \%$ \\
\hline
\end{tabular}

strong trend toward a survival benefit with pertuzumabtrastuzumab-docetaxel with an HR of 0.64 (95\% CI, 0.470.88 ) (fig. 4). The confirmatory overall survival analysis performed after 267 deaths and a median follow-up of 30 months in both arms confirmed a significant survival benefit for the combination of pertuzumab, trastuzumab and docetaxel $(\mathrm{HR}=0.66 ; 95 \% \mathrm{CI}, 0.52-0.84, \mathrm{p}=0.0008)$ [41]. There was also a significant increase in objective response rate from $69.3 \%$ in the control to $80.2 \%$ in the pertuzumab group $(\mathrm{p}=0.001)$.

The combination of pertuzumab and trastuzumab plus docetaxel increased rates of diarrhea, rash, mucosal inflammation, febrile neutropenia, and dry skin. These adverse events were primarily grades 1 or 2 and manageable $[39,40]$. The great majority of events (all grades and grades $\geq 3$ ) occurred during concomitant antibody and docetaxel administration with a substantial reduction in rates of the most common adverse events following discontinuation of docetaxel. After stopping administration of docetaxel febrile neutropenia was no longer reported in the placebo or the pertuzumab arm [42]. Importantly, there was no increase in cardiac adverse events or LVSD with the addition of pertuzumab (table 1). A univariate Cox regression analysis demonstrated that development of LVSD was associated with prior therapy with anthracyclines and prior radiotherapy. There was no significant association between prior trastuzumab therapy and development of LVSD [43].

Quality of life was similar for both study arms. An exploratory analysis showed that the combination of pertuzumab, trastuzumab and docetaxel was associated with a substantial delay in the time to onset of specific breast cancer symptoms with a median time to deterioration of symptoms of 18.3 weeks for placebo, trastuzumab and docetaxel compared to 26.7 weeks for the pertuzumab, trastuzumab and docetaxel $\operatorname{arm}(\mathrm{HR}=0.77 ; 95 \%$ CI 0.64-0.93, $\mathrm{p}=0.0061)$ [44]

\section{Pertuzumab - Ongoing and Planned Studies}

There are a number of ongoing and planned studies aimed at evaluating the combination of pertuzumab and trastuzumab in different settings of HER2-positive breast cancer. The ongoing randomized phase II study PHEREXA is evaluating the efficacy and safety of trastuzumab plus capecitabine with

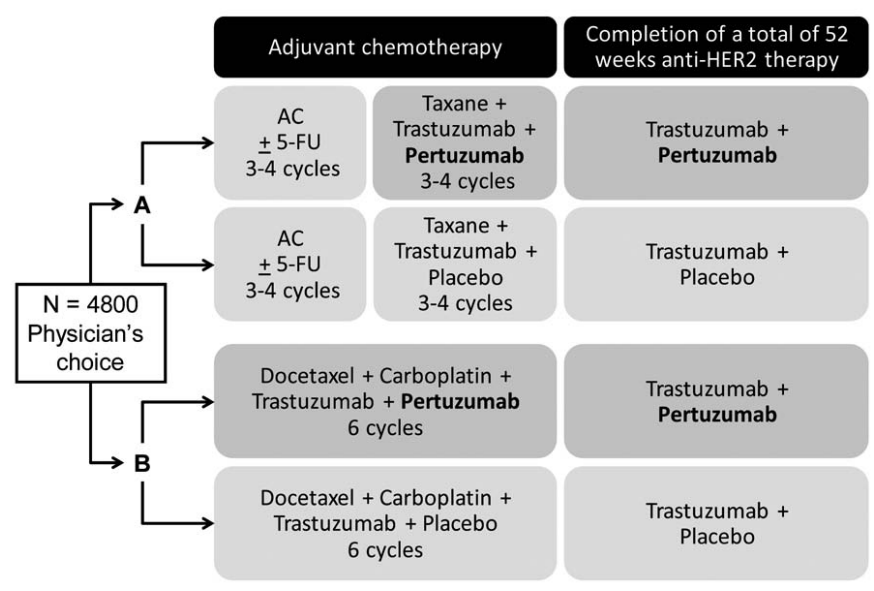

Fig. 5. APHINITY: Study design. Trastuzumab loading dose at first cycle $8 \mathrm{mg} / \mathrm{kg}$, dose of $6 \mathrm{mg} / \mathrm{kg}$ 3-weekly in subsequent cycles. Pertuzumab loading dose of $840 \mathrm{mg}$ i.v., dose of $420 \mathrm{mg}$ i.v. 3-weekly in subsequent cycles. Placebo i.v. 3 weekly. A limited number of standard anthracycline or non-anthracycline $(\mathrm{TCH})$ regimens are allowed.

or without the addition of pertuzumab in the treatment of patients with HER2-positive MBC who progressed after 1 line of trastuzumab-based therapy. The planned randomized phase II study VELVET will evaluate how the addition of pertuzumab affects efficacy and safety of the combination of trastuzumab and vinorelbine as a first- or second-line treatment of patients with HER2-positive MBC.

The German Breast Group started the randomized phase III trial, GEPARSepto, comparing neoadjuvant paclitaxel to nanoparticle albumin-bound (nab)-paclitaxel and evaluating dual inhibition with trastuzumab plus pertuzumab in HER2-positive patients. Moreover, surrogate markers for early response to dual inhibition with trastuzumab and pertuzumab will also be evaluated in the WSG ADAPT Trial (www.wsg-online.com). Finally, the large international phase III study APHINITY (fig. 5) will evaluate the added value of pertuzumab for the adjuvant treatment of patients with HER2-positive early breast cancer. In this trial, following an upfront physicians' choice between an anthracycline-based and a non-anthracycline platinum-based adjuvant chemotherapy regimen, patients will be randomized to standard adjuvant trastuzumab plus pertuzumab or adjuvant trastuzumab plus placebo. 


\section{Conclusions}

Due to their different mechanisms of action, pertuzumab and trastuzumab act in a complementary fashion and provide a more complete blockade of HER2-mediated signal transduction than either agent alone. Proof of principle of the efficacy of dual HER2 inhibition with the dimerization inhibitor pertuzumab and trastuzumab has been provided by the data of the pivotal phase III study CLEOPATRA, which has shown a significant and clinically relevant prolongation of PFS by 6.1 months with the addition of pertuzumab in first-line HER2-positive MBC, resulting in a significant survival benefit. Most importantly, several studies demonstrated that adding pertuzumab is not associated with increased cardiac toxicity. Currently, the combination of both antibodies is being explored in the palliative setting as well as in the treatment of early HER2-positive breast cancer. Dual HER2 inhibition with pertuzumab and trastuzumab may change clinical practice in HER2-positive first-line MBC, and beyond, in HER2positive breast cancer.

\section{Acknowledgements}

The authors are grateful to Dr. Susanne Hell for her medical writing and editorial support. Funding for this support was provided by Roche AG, Germany. The draft manuscript was reviewed and revised by the first and the senior author (N.H., C.J.). The final manuscript was reviewed, revised, and approved by all authors. The authors are solely responsible for the content of the manuscript with no restrictions set by the sponsor.

\section{Disclosure Statement}

The following conflicts of interest were disclosed: NH honoraria for consulting and lectures from Roche and GSK; MB participation in advisory boards for GSK, Astra Zeneca, Novartis, Pfizer, Sanofi-Aventis, Amgen, Roche; AR participation in an advisory board for Roche, AS honoraria for consulting and lectures from Roche; VM honoraria for lectures from Amgen, Celgene, Sanofi-Aventis, Pierre-Fabre, Roche and research funding from Roche; TF member of the advisory board of Roche; NM member of the advisory board of Roche; OG member of the advisory board and the speakers' bureau of Roche; CJ member of the advisory board and the speakers' bureau of Roche. IS and MU had no conflict of interest to declare with this publication.

\section{References}

1 Choritz H, Büsche G, Kreipe H, et al.: Quality assessment of HER2 testing by monitoring of positivity rates. Virchows Arch 2011;459:283-289.

2 Slamon DJ, Clark GM, Wong SG, et al.: Human breast cancer: Correlation of relapse and surviva with amplification of the HER-2/neu oncogene. Science 1987;234:177-182.

$\checkmark 3$ Ross JS, Slodkowska EA, Symmans WF, et al.: The HER-2 receptor and breast cancer: Ten years of targeted anti-HER-2 therapy and personalized medicine. Oncologist 2009;14:320-368.

$\checkmark 4$ Piccart-Gebhart MJ, Procter M, Leyland-Jones B, et al.: Trastuzumab after adjuvant chemotherapy in HER2-positive breast cancer. N Engl J Med 2005;353:1659-1673.

5 Smith I, Procter M, Gelber R, et al.: Two-year follow-up of trastuzumab after adjuvant chemotherapy in HER2-positive breast cancer: a randomised controlled trial. Lancet 2007;369:29-36.

6 Gianni L, Goldhirsch A, Gelber RD, et al.: Update of the HERA trial and the role of 1 year trastuzumab as adjuvant therapy for breast cancer. Breast 2009;18 (suppl 1):abstract S25.

7 Romond EH, Perez EA, Bryant J, et al.: Trastuzumab plus adjuvant chemotherapy for operable HER2-positive breast cancer. N Engl J Med 2005;353:1673-1684.

8 Perez EA, Romond EH, Suman VJ, et al.: Updated results of the combined analysis of NCCTG N9831 and NSABP B-31 adjuvant chemotherapy with/without trastuzumab in patients with HER2positive breast cancer. Proc Am Soc Clin Oncol 2007;25:abstract 512.

$>9$ Slamon D, Eiermann W, Robert N, et al.: Adjuvant trastuzumab in HER2-positive breast cancer N Engl J Med 2011;365:1273-1283.

10 Slamon DJ, Leyland-Jones B, Shak S, et al.: Use of chemotherapy plus a monoclonal antibody against HER2 for metastatic breast cancer that overexpresses HER2. N Engl J Med 2001;344:783-792.

11 Dawood S, Broglio K, Buzdar AU, et al.: Prognosis of women with metastatic breast cancer by HER2 status and trastuzumab treatment: an institutionalbased review. J Clin Oncol 2010;28:92-98.

12 Blackwell KL, Burstein HJ, Storniolo AM, et al.: Randomized study of Lapatinib alone or in combination with trastuzumab in women with ErbB2positive, trastuzumab-refractory metastatic breast cancer. J Clin Oncol 2010;28:1124-1130.

13 Franklin MC, Carey KD, Vajdos FF, et al.: Insights into ErbB signaling from the structure of the ErbB2-pertuzumab complex. Cancer Cell 2004; 5:317-328.

14 Yarden Y, Sliwkowski MX: Untangling the ErbB signalling network. Nat Rev Mol Cell Biol 2001;2:127-137.

15 Eccles SA: The epidermal growth factor receptor7Erb-B/HER family in normal and malignant breast biology. Int J Dev Biol 2011; 55:685-696.

16 Tzahar E, Waterman H, Chen X, et al.: A hierarchical network of interreceptor interactions determines signal transduction by Neu differentiation factor/neuregulin and epidermal growth factor. Mol Cell Biol 1996;16:5276-5287.

17 Citri A, Skaria KB, Yarden Y: The deaf and the dumb: The biology of ErbB-2 and ErbB-3. Exp Cell Res 2003;284:54-65.

18 Lee-Hoeflich ST, Crocker L, Yao E, et al.: A central role for HER3 in HER2-amplified breast cancer: Implications for targeted therapy. Cancer Res 2008; 68:5878-5887.

19 Sergina NV, Rausch M, Wang D, et al.: Escape from HER-family tyrosine kinase inhibitor therapy by the kinase-inactive HER3. Nature 2007;445:437-441.

20 Baselga J: A new anti-ErbB2 strategy in the treatment of cancer: Prevention of ligand-dependent ErbB2 receptor heterodimerization. Cancer Cell 2002; 2:93-95.

21 Agus DB, Akita RW, Fox WD, et al.: Targeting ligand-activated ErbB2 signaling inhibits breast and prostate tumor growth. Cancer Cell 2002;2:127-137.
22 Scheuer W, Friess T, Burtscher H, et al.: Strongly enhanced antitumor activity of trastuzumab and pertuzumab combination treatment on HER2-positive human xenograft tumor models. Cancer Res 2009; 69:9330-9336.

23 Molina MA, Codony-Servat J, Albanell J, et al.: Trastuzumab (Herceptin), a humanized anti-HER2 receptor monoclonal antibody, inhibits basal and activated HER2 ectodomain cleavage in breast cancer cells. Cancer Res 2001;61:4744-4749.

24 Nahta R, Hung MC, Esteva FJ: The HER-2-targeting antibodies trastuzumab and pertuzmab synergistically inhibit the survival of breast cancer cells. Cancer Res 2004;64:2343-2346.

25 Adams CW, Allison DE, Flagella K, et al.: Humanization of a recombinant monoclonal antibody to produce a therapeutic HER dimerization inhibitor, pertuzumab. Cancer Immunol Immunother 2006;55:717-727.

26 Agus DB, Gordon MS, Taylor C, et al.: Phase I clinical study of pertuzumab, a novel HER dimerization inhibitor, in patients with advanced cancer. J Clin Oncol 2005;23:2534-2543.

27 Yamamoto N, Yamada Y, Fujiwara Y, et al.: Phase I and pharmacokinetic study of HER2-targeted rhuMAb 2C4 (Pertuzumab, RO4368451) in Japanese patients with solid tumours. Jpn J Clin Oncol 2009;39:260-266.

28 Agus DB, Sweeney CJ, Morris MJ, et al.: Efficacy and safety of single-agent pertuzumab (rhuMAb 2C4), a human epidermal growth factor receptor dimerization inhibitor, in castration-resistant prostate cancer after progression from taxane-based therapy. J Clin Oncol 2007;25:675-681.

29 De Bono JS, Bellmunt J, Attard G, et al.: Openlabel phase II study evaluating the efficacy and safety of two doses of pertuzumab in castrate chemotherapy-naive patients with hormone-refractory prostate cancer. J Clin Oncol 2007;25:257-262.

30 Herbst RS, Davies AM, Natale RB, et al.: Efficacy and safety of single-agent pertuzumab, a human epidermal receptor dimerization inhibitor, in pa- 
tients with non small cell lung cancer. Clin Cancer Res 2007;13:6175-6181.

-31 Gordon MS, Matei D, Aghajanian C, et al.: Clinical activity of pertuzumab (rhuMAb 2C4), a HER dimerization inhibitor, in advanced ovarian cancer: Potential predictive relationship with tumour HER2 activation status. J Clin Oncol 2006;24:4324 4332.

32 Gianni L, lladó A, Bianchi G, et al.: Open-label, Phase II, multicenter, randomised study of the efficacy and safety of two dose levels of pertuzumab, a HER2 dimerization inhibitor, in patients with HER2-negative metastatic breast cancer. J Clin Oncol 2010;28:1131-1137.

33 Lenihan D, Suter T, Brammer M, et al.: Pooled analysis of cardiac safety in patients with cancer treated with pertuzumab. Ann Oncol 2012, 23:791800.

-34 Ng CM, Lum BL, Gimenez V, et al.: Rationale for fixed dosing of pertuzumab in cancer patients based on population pharmacokinetic analysis. Pharm Res. 2006;23:1275-1284.

\35 Baselga J, Gelmon KA, Verma S, et al.: Phase II trial of pertuzumab and trastuzumab in patients with human epidermal growth factor receptor 2-positive metastatic breast cancer that had progressed during prior trastuzumab therapy. J Clin Oncol 2010;28:1138-1144.

36 Gianni L, Pienkowski T, Im Y-H, et al.: Efficacy and safety of neoadjuvant pertuzumab and trastu- zumab in women with locally advanced, inflammatory, or early HER2-positive breast cancer (NeoSphere): A randomised multicentre, open-label, phase 2 trial. Lancet Oncol 2012;13:25-32.

37 Gianni L, Bianchini G, Kiermaier A, et al.: Neoadjuvant pertuzumab $(\mathrm{P})$ and trastuzumab $(\mathrm{H})$ : Biomarker analyses of a 4-arm randomized phase II study (NeoSphere) in patients (pts) with HER2positive breast cancer (BC). Abstracts of the 34th Annual CTCR-AACR San Antonio Breast Cancer Symposium 6-10 December 2011. Cancer Res 2011;71, suppl 3:S5-1.

38 Schneeweiss A, Chia S, Hickish T, et al.: Neoadjuvant pertuzumab and trastuzumab concurrent or sequential with an anthracycline-containing or concurrent with an anthracycline-free standard regimen: a randomized phase II study (TRYPHAENA). Abstracts of the 34th Annual CTRC-AACR San Antonio Breast Cancer Symposium, 6-10 December 2011. Cancer Res 2011;71, Suppl 3:S5-6.

39 Baselga J, Cortes J, Kim S-B, et al.: Pertuzumab plus trastuzumab plus docetaxel for metastatic breast cancer. N Engl J Med 2012;366:109-119.

40 Baselga J, Kim SB, Alm S, et al. Phase III, randomized, double-blind, placebo-controlled registration trial to evaluate the efficacy and safety of placebo + trastuzumab + docetaxel vs.pertuzumab + trastuzumab + docetaxel in patients with previously untreated HER2-positive metastatic breast cancer (CLEOPATRA). Abstracts of the 34th Annual
CTRC-AACR San Antonio Breast Cancer Symposium, 6-10 December 2011. Cancer Res 2011;71, Suppl 3:S5-5.

41 Swain S, Kim S-B, Cortés J, et al: Confirmatory overall survival analysis of CLEOPATRA A randomized, double-blind, placebo-controlled Phase III study with pertuzumab, trastuzumab, and docetaxel in patients with HER2-positive first-line MBC. Abstracts of the 35th Annual CTRC-AACR San Antonio Breast Cancer Symposium, 4-8 December 2012. Cancer Res 2012;72, Suppl 3:abstract P5-18-26.

42 Baselga J, Cortes J, Im S-A, et al.: Adverse events with pertuzumab and trastuzumab: Evolution during treatment with and without docetaxel in CLEOPATRA. J Clin Oncol 2012;30, Suppl:abstract 597.

43 Ewer MS, Baselga J, Clark E, et al.: Cardiac tolerability of pertuzumab + trastuzumab + docetaxel in patients with HER2-positive metastatic breast cancer in the CLEOPATRA study. J Clin Oncol 2012;30, Suppl:abstract 533

44 Cortés J, Baselga J, Im Y-H, et al.: Quality of life assessment in CELOPATRA, a phase III study combining pertuzumab with trastuzumab and docetaxel in metastatic breast cancer. J Clin Oncol 2012;30, Suppl:abstract 598. 